\title{
THE ROLE OF INFLAMMATION MODULATION IN DENTAL PULP REGENERATION
}

\author{
S.H. Zaky ${ }^{1,2,3}$, M. Shehabeldin ${ }^{1,2}$, H. Ray ${ }^{1,4,5}$ and C. Sfeir ${ }^{1,4,6, *}$ \\ ${ }^{1}$ Centre for Craniofacial Regeneration, School of Dental Medicine, University of Pittsburgh, \\ Pittsburgh, PA, USA \\ ${ }^{2}$ Department of Oral Biology, School of Dental Medicine, University of Pittsburgh, Pittsburgh, PA, USA \\ ${ }^{3}$ Department of Restorative Dentistry, School of Dental Medicine, University of Pittsburgh, \\ Pittsburgh, PA, USA \\ ${ }^{4}$ McGowan Institute for Regenerative Medicine, University of Pittsburgh, Pittsburgh, PA, USA \\ ${ }^{5}$ Department of Endodontics, School of Dental Medicine, University of Pittsburgh, Pittsburgh, PA, USA \\ ${ }^{6}$ Department of Periodontics, School of Dental Medicine, University of Pittsburgh, Pittsburgh, PA, USA
}

\begin{abstract}
A vital and healthy dental pulp (DP) is required for teeth to remain functional throughout a lifespan. Appreciating its value for the tooth, the regeneration of the DP is a highly researched goal. While inflammation of the DP marks the beginning of an eventual necrosis, it is also the prerequisite for the regenerative events of neovascularisation, stem cell mobilisation and reparative dentine deposition. In the light of a pro-regenerative inflammatory process, the present review discusses the role of the macrophage population shift from pro- to anti-inflammatory in reversible versus irreversible pulpitis, while also analysing the overlooked contribution of pulp innervation and locally derived neuropeptides to the process. Then, the currently practiced (pulp capping and revascularisation) and researched (cell transplantation and cell homing) approaches for DP regeneration are discussed. Focusing on the role of cell homing in modulating inflammation, some potential strategies are highlighted to harness the inflammatory process for DP regeneration, mainly by reversing inflammation through macrophage induction. Next, some potential clinical applications are discussed - especially with capping materials - that could boost macrophage polarisation and complement system activation. Finally, current challenges facing the regeneration of the DP are presented, while underlining the importance of promoting an anti-inflammatory environment conducive to a regenerative process.
\end{abstract}

Keywords: Dental pulp, inflammation modulation, regeneration, pro-inflammtory cytokines.

*Address for correspondence: C. Sfeir, Centre for Craniofacial Regeneration, University of Pittsburgh, 501 Salk Pavilion, 335 Sutherland Drive, Pittsburgh, PA, 15213, USA.

Telephone number: +14126481949 Fax number: +1 4126246685 Email:csfeir@pitt.edu

Copyright policy: This article is distributed in accordance with Creative Commons Attribution Licence (http://creativecommons.org/licenses/by-sa/4.0/).

\section{List of Abbreviations}

BMP-2

CCL2

$\mathrm{CD}$

CGRP

DP

DPSCs

ECM

IFN

IL

NGF

PLA

RCT bone morphogenetic protein-2

$\mathrm{C}-\mathrm{C}$ motif chemokine ligand 2

cluster of differentiation

calcitonin gene-related peptide

dental pulp

DP stromal cells

extracellular matrix

interferon

interleukin

nerve growth factor

polylactic acid

root canal treatment

\section{Introduction}

The DP occupies the core of the tooth and is the reason for its vitality. Although the DP might not be considered to contribute directly to tooth function - mastication, speech, aesthetic - a vital and healthy pulp is the reason why teeth remain functional throughout a lifetime. In fact, RCT - a tooth devitalising procedure and ironically also known as "tooth-saving" - is a radical treatment that results in the eventual loss or extraction of the tooth due to reinfection, secondary decay and leakage around crown margins and periodontal involvement (Friedman and Mor, 2004; Salehrabi and Rotstein, 2004; Siqueira, 2001). 
In 1951, Markley sensibly defined and approached dental decay [the most prevalent human disease (Cooper et al., 2010; Heng, 2016)]: “The loss of even a part of a human tooth should be considered a serious injury and that dentistry's goal should be to preserve healthy, natural tooth structure" (Markley, 1951). This, together with the understanding of the toothcore's invaluable role in maintaining lifelong tooth function, made DP regeneration a highly researched goal.

Root canal treatment is an appealing alternative to tooth extraction, however, not without limitations. After RCT, the physico-mechanical properties of the tooth are altered and it becomes more prone to fracture (Vongsavan et al., 2000). Even with a protective covering restoration, micro leakage is highly prevalent, resulting in periodontal involvement and undetected decay due to its hidden location under the covering crown together with the lack of the alarming pain reaction (Lucarotti, 2003; Ray and Trope, 1995). Yet, more importantly, tooth devitalisation eliminates the innate immune and repair responses of a vital pulp (Farges et al., 2015; Hahn and Liewehr, 2007). This inflammationmodulated immune response, orchestrated by pulp fibroblasts, odontoblasts, endothelial cells and even nerve fibres (Zhan et al., 2020), plays a key role in fighting against caries invasion as well as secretion of tertiary and reparative dentine (Farges et al., 2015).

The present review highlights the role of inflammation in DP repair and regeneration, while underlining the potential clinical approaches that could reverse DP inflammation, mainly by macrophage induction, towards pulp regeneration.

\section{The role of inflammation in pulpal injury and repair}

Within the dynamic DP tissue, homeostasis is disrupted by several pathologies, such as bacterial toxins or actual bacterial invasion, thermal or electrical insults (galvanism) (Certosimo and O'Connor, 1996). With tooth decay progression and before bacteria reach the DP, the diffusion of bacterial toxins into dentine tubules to the pulp-dentine interface triggers inflammatory and immune events in the underlying DP. Namely, antigen-presenting dendritic cells strategically migrate to the odontoblast layer facing the invasion, followed by accumulation of T-lymphocytes, macrophages, neutrophils and B-lymphocytes. Reversible pulpitis develops while bacterial invasion and toxins concentration are limited and inflammation is mild (Goldberg et al., 2008). In fact, the dentine-pulp complex responds to insults in a dose-effect manner, with low insult levels reversed to promote regeneration, while intense immune reaction irreversibly initiates tissue necrosis (Cooper et al., 2010). With fast, severe or longlasting (chronic) insults, odontoblasts are too slow to secrete reactionary dentine and, with further toxin accumulation and invasion of the DP, irreversible pulpitis is initiated followed by necrosis, root canal infection and periapical disease.

Before tissue necrosis, prostaglandin-mediated pain impulses are manifested clinically as a pulp reaction through the transmission carried by sensory nerve endings that, along with the sympathetic nerve endings, also play a role in regulating the DP inflammatory process by neuropeptide secretion targeting membrane bound receptors located on different pulp structures (Zhan et al., 2020). Neuropeptides, such as substance $P$, stimulate the production of pro-inflammatory cytokines from human DP fibroblasts; while CGRP is associated with immunosuppression and increased expression of BMP-2, promoting dentineogenesis within the DP (Calland et al., 1997). CGRP was, in fact, reported to promote an osteodentine bridge following pulpotomy (Kline and Yu, 2009). Membrane-bound receptors for functional molecules from dental nerves are expressed on odontoblasts, fibroblasts, inflammatory cells and endothelial cells of capillaries, indicating a bidirectional cross-talks between these structures and nerve endings. Besides supporting the odontoblastic receptor theory of dentine sensitivity (Zhan et al., 2020), such intimate anatomical and functional interaction between pulpal nerve endings and other pulpal structures strongly suggests their intertwining functional relationship in all DP events, including inflammation and regeneration.

\section{A mindset shift by a cell population shift: reversible versus irreversible pulpitis}

For years, inflammation was a term describing a vicious event of pain, redness and hotness prior to tissue necrosis and was viewed as antagonistic to regeneration. Currently, within an ascending learning curve, it is known that the inflammatory reaction is a prerequisite to initiate the reparative function of progenitor cells implicated in tissue restoration (Goldberg et al., 2008). Basically, a (pro-) inflammatory phase, when tissues are lost, timely shifts to an anti-inflammatory phase, characterised by proliferation and differentiation of stem cells for healing and regeneration, indicating the significant interrelationship between the inflammatory and regeneration processes (Cooper et al., 2010).

During inflammation followed by wound healing and tissue remodelling, the cellular response especially that of macrophages - is finely tuned (Roy, 2016). Following injuries - such as bacterial endotoxin invasion through dentineal tubules, deep cavity or crown preparation - a controlled and regulated inflammatory response cascades for pulp remodelling. However, if not controlled, for example in the case of bacterial invasion of the DP, a severe blow or long-standing insult, irreversible tissue damage takes place (Kawashima et al., 2005; Mutoh et al., 2009).

The correlation between macrophage population shift, known as polarisation, and tissue remodelling 
is under extensive investigation (Mahmoudi et al., 2017; Yonehiro et al., 2012). During the inflammatory process, monocytes are recruited by neutrophils and, then, migrate to the injury site where they differentiate into macrophages (Swirski et al., 2009). Once recruited and differentiated, the macrophages generally attain M1 polarisation, a pro-inflammatory phenotype triggered by exposure to recruiting proinflammatory cytokines such as IFN- $\gamma$ (St Pierre and Tidball 1994; Tidball, 2005). In about $48 \mathrm{~h}$, M1 macrophages reach their maximum number. Synthesis of IL-10 and IL-4 recruits and shifts the population to the M2 immuno-regulatory and antiinflammatory phenotype (Collins and Grounds 2001; Villalta et al., 2009).

As reversible pulpitis is initiated and progresses, classically activated M1 macrophages mediate phagocytosis of bacteria, removal of dead cells and the initial exposure to the ECM-degradation products (Sicari et al., 2014). Once polarised to the M2 phenotype, macrophages release anti-inflammatory cytokines that signal tissue remodelling and repair (Gordon, 2003; Gordon and Martinez, 2010) (Fig. 1). As insult intensifies, interruption of the proinflammatory events take place: neutrophil and macrophage infiltration, polarisation or transition are halted and, hence, remodelling or regeneration processes (Tidball and Wehling-Henricks, 2007). The interruption of the regeneration process, in the case of a DP injury, is manifested as activated osteo/odontoclastogenesis, modified vascular endothelial permeability with concomitant bone and root resorption (Lara et al., 2003; Zaky et al., 2020). Targeting the sustainability of a reversible

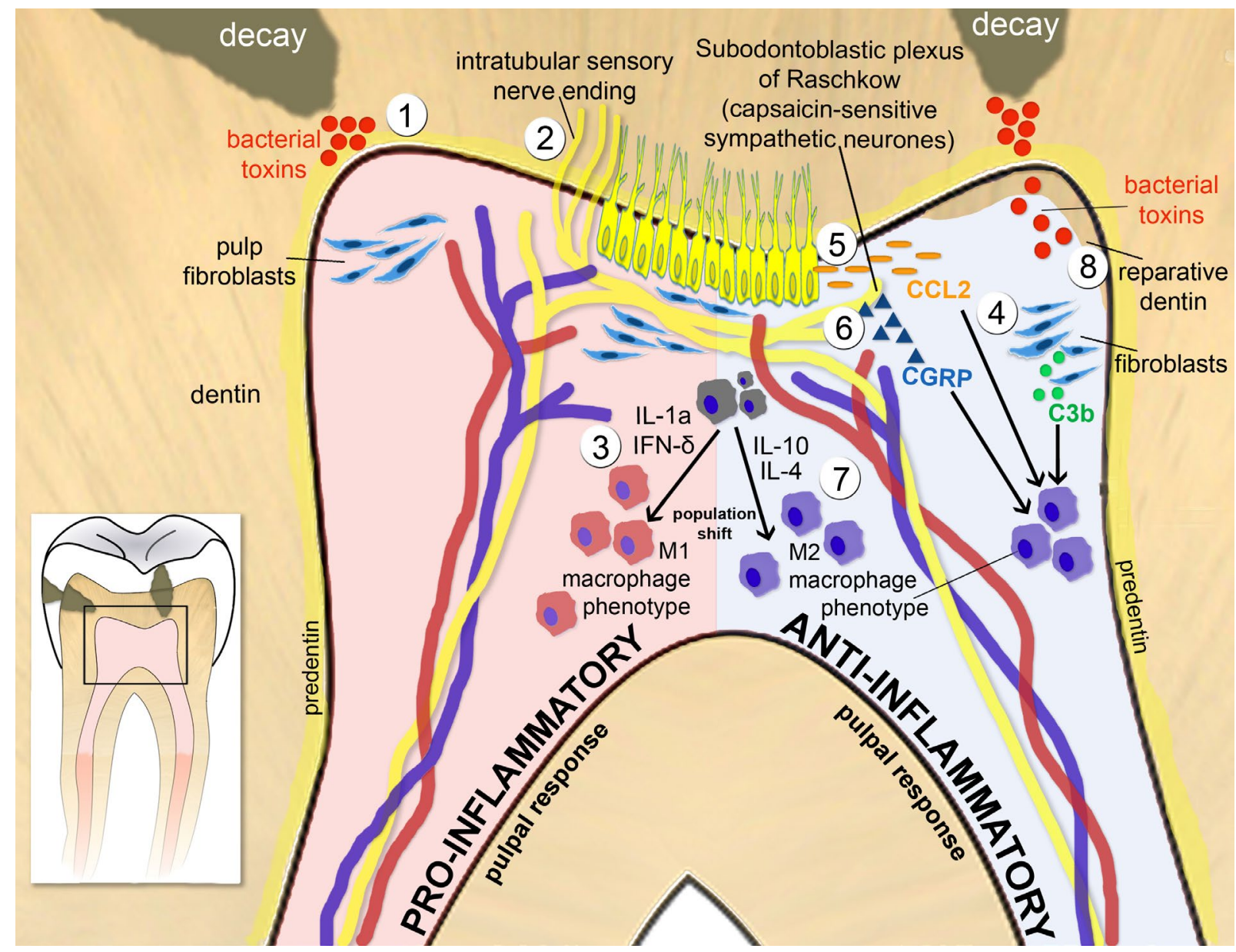

Fig. 1. Schematic drawing of pulpal events taking place in pro-inflammatory (left in red) and antiinflammatory (right in blue) responses and the potentially tailorable approaches to modulate inflammation to achieve regeneration. Numbers indicate separate events rather than an accurate chronological order. 1: bacterial toxins (noxious stimulus) are introduced in dentineal tubules. 2: intratubular sensory nerve endings are the first to receive the noxious stimulus. 3: extravasating macrophages are signalled to an M1 phenotype by IL-1a and IFN- $\delta$. 4: pulp fibroblasts activate C3b complement to mediate phagocytosis of bacteria toxins by M1 macrophages while contributing to their population shifts to an M2 phenotypes. As toxins advance within the dentineal tubules to the dental pulp, they stimulate a cascade of events that signal the anti-inflammatory shift to tissue remodelling and repair. 5: while still within the dentineal tubules, toxins stimulate odontoblasts to secrete CCL2. 6: reaching the dental pulp, toxins stimulate CGRP release from sympathetic nerve endings. 7: as the noxious stimulus subsides (decay removed), the macrophage population shifts to M2 phenotype by IL-10 and IL-4 action. 8: areas of reparative dentine are an indication of an anti-inflammatory, pro-regenerative homeostasis. 
pulpitis from a clinical standpoint would be crucial to interrupt the pro-inflammatory events by induction of the anti-inflammatory macrophages as early as possible to prevent the progression and accumulation of a reversible damage.

\section{The role of nerve endings and neuropeptides in anti-inflammatory macrophages induction}

Neurogenic inflammation describes the direct participation of dental innervation in the innate immune response (Cooper et al., 2010). From the subodontoblastic plexus of Rashkow in the coronal pulp, the sensory axons enter dentineal tubules to penetrate the dentine, whereas the sympathetic axons end in close proximity to odontoblast cell bodies (Ibuki et al., 1996; Shimeno et al., 2008). From within the tubules and receiving the bacterial toxin first-hand, the sensory group acts mainly in a pro-inflammatory manner initiating the pulpitis reaction. Progressing within the pulp, the sympathetic group, associated with the pulpal aspect of the odontoblastic layer, counteracts by an anti-inflammatory action. Such action includes the induction of anti-inflammatory macrophages by inhibition of cytokine IL-1a production, a bone-resorbing and a pro-inflammatory factor (Bletsa et al., 2004; Zhan et al., 2020).

NGF, a neuropeptide involved in the regulation of growth, maintenance, proliferation and survival of pulpal neurons, is highly expressed at the caries invasion site (Mitsiadis et al., 2017). This takes place either directly by upregulation and even redistribution of its receptors during pulpitis (Woodnutt et al., 2000) or indirectly by nerve endings outgrowth in response to human pulp fibroblast through complement system activation (Chmilewsky et al., 2016). As bacterial toxins progress towards the pulp, the upregulation of NGF signals a pulpal reparative process to reverse inflammation towards regeneration.

Another neuropeptide involved in neurogenic inflammation, CGRP, was found to promote odontoblast differentiation and maintain their function (Chiego et al., 1987). Together with pulpal Schwann cells, CGRP promotes macrophage population shift to the anti-inflammatory macrophage M2 phenotype to regulate the immune response (Caviedes-Bucheli et al., 2008). In fact, abundant colocalisation of Schwann cells and M2 macrophages is detected in the DP under deep active decay, with faint sensitivity and without episodes of spontaneous pain (Yoshiba et al., 2020).

\section{Regeneration in endodontics}

The history of root canal therapy date back to 1728 when extirpation of the DP, using a small pin, was described (Cruse and Bellizzi, 1980). Since then, manipulating inside the tooth, i.e. endodontics, was coined as a branch of practicing dentistry; while the evolving instrumentation, techniques and obturation materials have been successful to make RCT an efficient treatment with more than a $95 \%$ success rate (Naito, 2005). However, RCT is intrinsically a tooth devitalisation process whose success is judged solely by the asymptomatic retention of the tooth in place regardless of the ability of the pulp-dentine complex to react to its environment - a characteristic of a vital structure only. With that in mind, RCT is the resort when signs of irreversible pulpitis are confirmed; while approaches for vital pulp therapy are attempted to maintain the pulp vitality even with wavering reversibility. It is important to note that for $\mathrm{RCT}$, vital pulp therapy or any regenerative therapy, it is of global consensus that clearing off bacterial infection, within the pulp canals or from tooth decay, is the key for treatment success.

\section{Clinically practiced regenerative approaches}

It is out of awareness and appreciation of the DP tissue that resorting to RCT is not a decision to be taken lightly. While considering the younger pulp favoured response (Bansal and Bansal, 2011), several approaches have been researched, developed and clinically applied to attempt regenerating the DP at different extents of its ailment: from direct pulp capping covering a pinpoint exposure to vascularisation and re-innervation of a fully lost root canal system.

Direct pulp capping is a regenerative procedure and a clinical example of vital pulp therapy. When successful (93-100\%) (Akhlaghi and Khademi, 2015), direct pulp capping confirms that pulpitis is in a reversible phase. The inflammatory process, compartmentalised within the exposed coronal pulp, can still be reversed. Stem precursors and pericytes can be mobilised and differentiate into odontoblasts to construct a reparative dentine bridge (Wells et al., 2019).

Revascularisation is another regenerative approach, also referred to as a regenerative endodontic procedure, aiming at revitalising a necrotic immature permanent tooth. The necrotic tooth is debrided and disinfected with sodium hypochlorite and calcium hydroxide paste while the open apex allows stimulation of apical bleeding and invasion of a blood clot with all its healing properties, including stem cell colonisation from the apical papilla and neo-vascularisation. Some challenges exist, including patient compliance, overmanipulation and controlling infection. The success rate of this procedure ranges from bone healing, apex maturation (though unpredictable) and ultimately tooth positive response to vitality tests - indicating innervation (Ray et al., 2016; Staffoli et al., 2019)

\section{DP regeneration strategies in mature teeth}

In the case of adult mature teeth, pulp regeneration is primarily challenged by narrowing of the canal anatomy through dentineal sclerosis and the minimal 

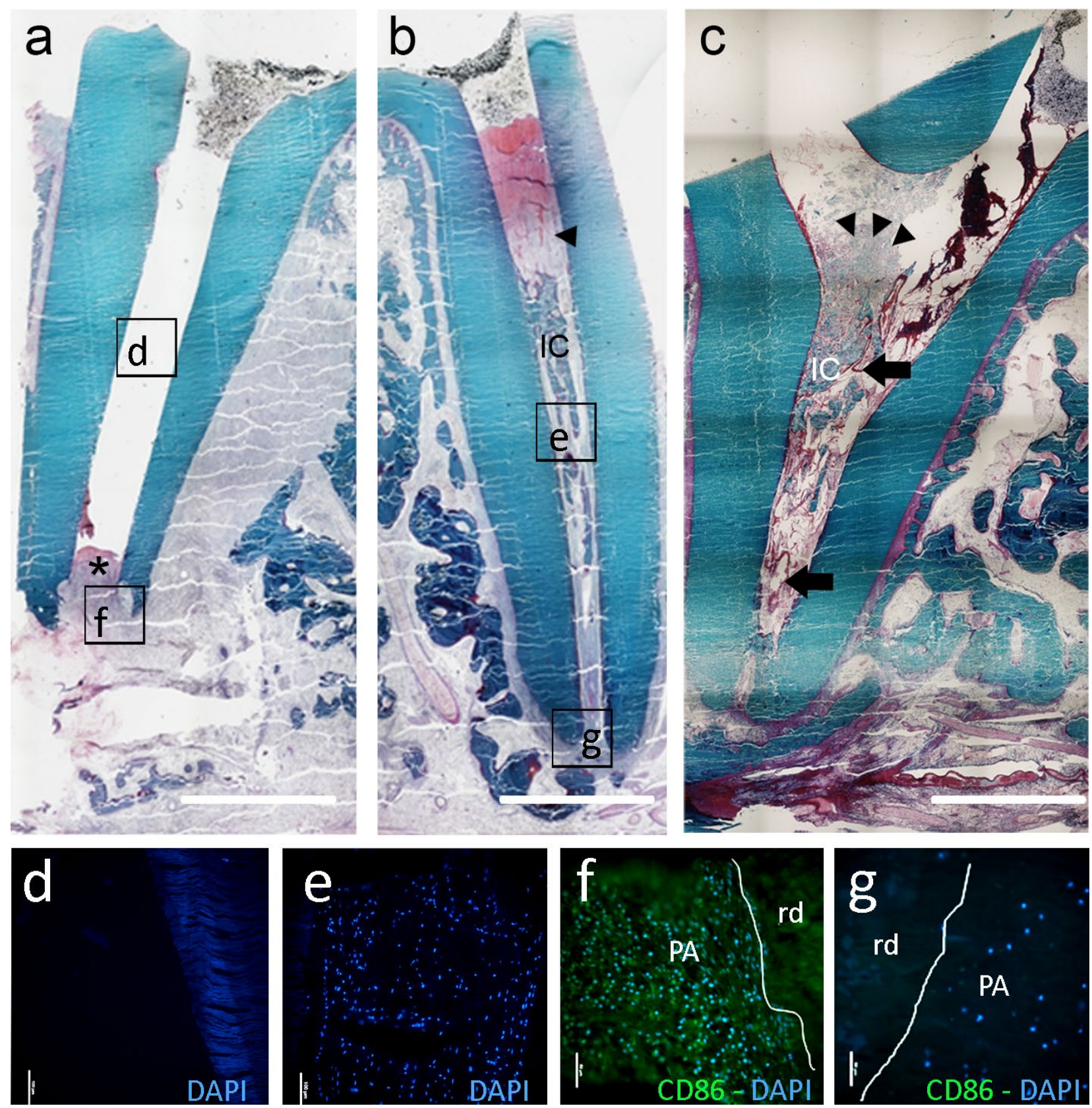

Fig. 2. Different regenerative outcomes in canine (beagles) root canal model according to the absence or presence of an inflammatory process. (a) With inflammation: root canal of beagle lower premolar manipulated without introduction of obturation material (only blood clot formation) while inflammation was induced periodically (*: inflammatory plug). (f) Immunofluorescence of CD86, an anti-M1 macrophages marker (Zaky et al., 2020). (d) No regeneration or cell infiltration is observed while signs of destruction are seen as bone and root dentine (rd) resorption. (b) Without inflammation: blood-clot-filled root canal without periapical inflammation (g: CD86 negative) showing regenerative process and cell infiltration (e and arrowheads) are observed without bone or root resorption. (c) Inflammation modulation: Swine ECMfilled root canal shows a regenerative process with cell infiltration (arrowheads) and more vascularisation (arrows) (Alqahtani et al., 2018). Although ectopic, bone/cementum-like intracanal calcifications (IC) within a remodelling pulp are a common observation in pulp regeneration literature and are considered signs of an active regenerative process (Alqahtani et al., 2018; Skoglund and Tronstad, 1981; Zaky et al., 2020). Hence, it is suggested to include cementogenesis inhibitor molecules while attempting pulp regeneration procedures by periapical cells homing (Zizka and Sedy, 2017). (a-c) Scale bars: $250 \mu$ m; (d,e) scale bars: $100 \mu \mathrm{m} ;(\mathbf{f}, \mathbf{g})$ scale bars: $50 \mu \mathrm{m}$. PA: periapical tissues. 
opening of the anatomic apical foramen - challenging the development of an anastomosing blood supply with the alveolar vessels (Huang et al., 2020). Pulp regeneration in mature teeth is attempted by two approaches: cell transplantation and cell homing, both equally challenging.

Cell transplantation involves delivering of donor cells. Several animal studies with transplanted cells have reported pulp regeneration with formation of an odontoblastic layer, blood vessels and evidence of neuronal regeneration employing DPSCs; either as a total population (Souron et al., 2014) or as an enriched subset (Iohara et al., 2009) in pulpotomy or full pulpectomy models (Iohara et al., 2020). To date, few human clinical trials report such promising results (Nakashima et al., 2017). Yet, controversy still exists as to whether these protocols are ready to replace conventional endodontic treatment procedures.

Cell homing is defined as the active recruitment of stem/progenitor endogenous cells into an anatomical compartment (Laird et al., 2008). Studies attempting DP regeneration by cell homing also report promising results in terms of development of pulp-like tissue that develops from recruited periapical/periodontal cells through a manipulated and widened apical foramen to colonise the root canals up to the pulp chamber (Alqahtani et al., 2018; Eramo et al., 2018; Huang et al., 2020). Interestingly, a blood clot formation within the debrided root canal and chamber is enough to support the regeneration process that occurs regardless of the different types of inserted scaffolds (Zaky et al., 2020) (Fig. 2).

While both approaches seem promising, the cell homing approach clearly circumvents, from a clinical standpoint, many of the challenges associated with cell transplantation. Undoubtedly, an acellular chair-side ready approach bypasses the hurdle of immunogenicity and the complexity of a substantial ex vivo cell manipulation and culture, hence is safer, more time and cost effective and more clinically applicable. Besides and more importantly, tissue regeneration by cell trafficking warrants the recruitment of all subsets of host cell populations and native growth factors to repopulate the defect and re-establish self-renewing stem-cell niches in a process mirroring physiological tissue remodelling and lifetime tissue turnover (Zaky and Cancedda, 2009).

\section{Cell homing and inflammation modulation}

Regeneration by cell homing, also including revascularisation techniques in endodontic regenerative therapy, occurs primarily by the periapical cell populations trafficking trough the root apex. These populations, later engulfed in a clot of red blood cells and platelets, include lymphocytes, endothelial cells, neuronal precursors, fibroblasts, monocytes (originating from bone marrow and differentiating into macrophages) and progenitor/ stem cells mobilised as pericytes from the blood vessel walls. All together, along with cytokines and growth factors in the specific proportions, offer the favourable cocktail for wound healing in general terms and specifically for pulp-like tissue development when within dentineal walls.

When it comes to regeneration, the role of macrophages in the process is key. Depending on the intensity of the bacterial infection or the extent of periapical instrumentation, cytokines trigger macrophagespolarisation from M1 proinflammatory to M2 anti-inflammatory phenotype to phagocytise bacteria and dead cells and signal a regeneration process (Sicari et al., 2014). In fact, with extensive periapical inflammation and M1 macrophage colonisation, dentine and root resorption are observed with no signs of regeneration within debrided root canals. In contrast, tissues show signs of remodelling and neovascularisation within the debrided pulp space when the periapex shows no signs of inflammation (Zaky et al., 2020).

Interestingly, the current literature suggests that DP fibroblasts play an important role in inflammation modulation by local activation of the complement system. Bacterial endotoxins stimulate DP fibroblasts to signal the secretion of the antimicrobial $\mathrm{C} 3 \mathrm{~b}$ complement not only to fight bacterial invasion (Le Fournis et al., 2019) but also to participate in the macrophage population shift from M1 to M2 macrophages (Bohlson et al., 2014) (Fig. 1).

\section{Harnessing the inflammatory process and macrophage induction for DP regeneration}

Taming the inflammatory process towards regeneration primarily involves the modulation of the process to sustain its anti-inflammatory phase over its pro-inflammatory phase. As macrophages are the main key players in this phase shift, the creation of a long-standing reversible pulpitis would be accomplished by induction of the M2 macrophages. Clinically, among several suggested approaches to modulate the inflammatory process - and the macrophages' shift to enhance regeneration - are the employment of decellularised ECM and the introduction of modulation molecules (Agrawal et al., 2010; Badylak et al., 2011; Frantz et al., 2010).

Although a blood clot formed by the bleeding within the wounded tissues contains sufficient recruited elements for regeneration (Lehwald et al., 2020; Radke et al., 2018), Alqahtani et al. (2018) suggested an approach to modulate the inflammation process within the blood clot. The approach employs decellularised DP ECM to be introduced into a debrided root canal while the blood clot forms after canal manipulation. Such an approach has a twofold benefit: i) the introduction of the ECM with its constitutional trophic factors into the root canal space triggers periapical progenitor/stem cell trafficking for the regeneration process; ii) the ECM also plays 
a role in inflammation modulation by triggering an anti-inflammatory host response that favours pulp regeneration (Agrawal et al., 2010; Badylak et al., 2011). The ECM would promote inflammatory modulation and create an antibacterial environment that supports DP regeneration (Fig. 2c) to reach homeostasis in the root canal and periapical tissues. The remodelling by ECM scaffolds is also attributed to the macrophage by-products released upon ECM digestion (Gordon and Taylor, 2005; Mantovani et al., 2004; Valentin et al., 2009).

Since macrophage modulation and polarisation play a crucial role in pulpal and periapical inflammation, it is intuitive to attempt DP regeneration by using macrophage-mobilising molecules to harness the inflammatory process for pulp regeneration. CGRP, a neuropeptide secreted by capsaicin-sensitive neurons in the DP, is known to promote proliferation of endothelial cells and pulpal fibroblasts and to increase DP vascular permeability (Bongenhielm et al., 1995; Zhan et al., 2020). CGRP is also a regulator of the immune response by acting as a signalling molecule for the M2 anti-inflammatory macrophage phenotype (Caviedes-Bucheli et al., 2008). CGRP has been reported to have a protective role in the initial phase of apical periodontitis (Austah et al., 2016).

Another molecule is CCL2, secreted by odontoblasts and diffused into the sub-odontoblast pulp area where they activate and mobilise various populations of immune cells (Farges et al., 2015). CCL2 has been shown to favour M2 macrophage polarisation (Zhuang et al., 2019) and to play a key role in the recruitment of circulating blood dendritic cells and their migration through the endothelial barrier (Goldberg et al., 2008) (Fig.1).

\section{Potential clinical application}

Clinically, it is crucial to narrow down to the inflammation modulation molecule that, upon delivery in contact or within the DP, can modulate and reverse the inflammation while signalling cell homing and differentiation. Such a molecule or combinations of molecules could be case-specific, depending on the treatment modalities needed to address a certain underlying pathosis, while considering the extent of irreversible damage to DP tissues.

The polarisation of the macrophage population from M1 pro-inflammatory to M2 anti-inflammatory phenotypes would be the primary target of cytokines' application to create a pro-regenerative environment (Colombo et al., 2014). CCL2 and CGRP molecules, among others, such as hypoxia-inducing factor (Colombo et al., 2020) and NGF neuropeptide (Mitsiadis et al., 2017), have the potential to leverage established regenerative applications such as direct pulp capping and revascularisation. These approaches rely on delivering a biocompatible, antibacterial, $\mathrm{Ca}^{2+}$-releasing material that mobilises bioactive molecules from the dentine (Cooper et al., 2010). While some pulp-capping materials have been reported to have inflammation-modulating effects (Giraud et al., 2019), their functionalisation with modulating molecules could boost their regenerative properties in terms of macrophage polarisation and complement system activation. Arguably, adding inflammation-modulating molecules to the capping materials could even open new horizons for mature teeth pulpotomy, currently approached as a provisional rather than a full alternative for RCT (Li et al., 2019; Sadaf, 2020).

Practically, the clinical delivery of these molecules would depend on their application modality, either as a single or cocktail combination. The relevant carrying vehicles currently researched both in vitro and in animal models are hydrogels (Colombo et al., 2020), polymeric scaffolds (Soares et al., 2018), PLA microparticles (Zhuang et al., 2019), nano-structured biomaterial (Keller et al., 2015) and collagen, among many others (Colombo et al., 2014). ECM application could also represent the delivery of a naturally occurring combination of inflammation-mediating cytokines (Alqahtani et al., 2018).

\section{Challenges and closing remarks}

With the different approaches and strategies for DP regeneration, it is clear that clinicians and researchers are still at the experimentation phase and far from offering a feasible clinical alternative to the current devitalising RCT. A considerable part of the lag could be attributed to the limited understanding of pulp biology, together with the absence of precise diagnostic tools that can indicate the progression or rather the reversibility of the pulp condition early enough (Duncan and Cooper, 2020).

With all the relatively advanced understanding of tooth development and its intricate biology, all current pulp regeneration approaches are attempting dentine and pulp regeneration in ways that do not mimic their original development. Throughout the epithelium-mesenchymal interaction, the pulp tissue develops simultaneously with and within the dentine while the dentine-secreting odontoblasts and their accompanying nerve endings recede along the maturing and mineralising dentine tissue pulpal surface from the dentineoenamel junction towards the pulp core (Farges et al., 2015; Zhan et al., 2020). As the fully developed tooth body has lost its soft tissue core, there is an attempt to re-generate the missing pulp tissue independently and in the absence of the timely orchestrated tooth development signalling molecules. Despite the observed potential of many approaches, among which inflammation modulation, this could be not the right way to proceed.

Compared to connective tissue maturation and neovascularisation, the regeneration of neural tissue remains the most challenging part. A fully restored 
and functional DP would need to acquire nerve regeneration in neural hierarchy and plexuses, not only to regain the alarming sensation of pain but primarily to regain its function in regulating inflammation and repair (Zhan et al., 2020).

As for the current different strategies for pulp regeneration, a key is, after dismissing the infection, promoting an anti-inflammatory environment that is conducive to tissue regeneration at the dentine-pulp interface. This is primarily achieved by introducing molecules and cytokines that can modulate the initial pro-inflammatory reaction and mobilise its cell population into anti-inflammatory reaction for a pro-regeneration process. Undoubtedly, unfolding research will enlighten the understanding of the molecular and cellular interactions that orchestrate this intricate inflammation-regeneration coupling to develop novel therapies for DP regeneration and regeneration of the complex tooth-organ as a whole.

\section{References}

Agrawal V, Johnson SA, Reing J, Zhang L, Tottey S, Wang G, Hirschi KK, Braunhut S, Gudas LJ, Badylak SF (2010) Epimorphic regeneration approach to tissue replacement in adult mammals. Proc Natl Acad Sci USA 107: 3351-3355.

Akhlaghi N, Khademi A (2015) Outcomes of vital pulp therapy in permanent teeth with different medicaments based on review of the literature. Dent Res J (Isfahan) 12: 406-417.

Alqahtani Q, Zaky SH, Patil A, Beniash E, Ray H, Sfeir C (2018) Decellularized swine dental pulp tissue for regenerative root canal therapy. J Dent Res 97: 1460-1467.

Austah ON, Ruparel NB, Henry MA, Fajardo RJ, Schmitz JE, Diogenes A (2016) Capsaicin-sensitive innervation modulates the development of apical periodontitis. J Endod 42: 1496-1502.

Badylak SF, Taylor D, Uygun K (2011) Wholeorgan tissue engineering: decellularization and recellularization of three-dimensional matrix scaffolds. Annu Rev Biomed Eng 13: 27-53.

Bansal R, Bansal R (2011) Regenerative endodontics: a state of the art. Indian J Dent Res 22: 122-131.

Bletsa A, Heyeraas KJ, Haug SR, Berggreen E (2004) Il-1 alpha and tnf-alpha expression in rat periapical lesions and dental pulp after unilateral sympathectomy. Neuroimmunomodulation 11: 376384.

Bohlson SS, O'Conner SD, Hulsebus HJ, Ho MM, Fraser DA (2014) Complement, c1q, and c1q-related molecules regulate macrophage polarization. Front Immunol 5: 402. DOI: 10.3389/fimmu.2014.00402.

Bongenhielm U, Haegerstrand A, Theodorsson E, Fried K (1995) Effects of neuropeptides on growth of cultivated rat molar pulp fibroblasts. Regul Pept 60: 91-98.
Calland JW, Harris SE, Carnes DL Jr (1997) Human pulp cells respond to calcitonin gene-related peptide in vitro. J Endod 23: 485-489.

Caviedes-Bucheli J, Moreno GC, Lopez MP, Bermeo-Noguera AM, Pacheco-Rodriguez G, Cuellar A, Munoz HR (2008) Calcitonin gene-related peptide receptor expression in alternatively activated monocytes/macrophages during irreversible pulpitis. J Endod 34: 945-949.

Certosimo AJ, O'Connor RP (1996) Oral electricity. Gen Dent 44: 324-326.

Chiego DJ, Jr., Avery JK, Klein RM (1987) Neuroregulation of protein synthesis in odontoblasts of the first molar of the rat after wounding. Cell Tissue Res 248: 119-123.

Chmilewsky F, Ayaz W, Appiah J, About I, Chung SH (2016) Nerve growth factor secretion from pulp fibroblasts is modulated by complement c5a receptor and implied in neurite outgrowth. Sci Rep 6: 31799. DOI: $10.1038 /$ srep31799.

Collins RA, Grounds MD (2001) The role of tumor necrosis factor-alpha (tnf-alpha) in skeletal muscle regeneration. Studies in tnf-alpha(-/-) and tnfalpha(-/-)/lt-alpha(-/-) mice. J Histochem Cytochem 49: 989-1001.

Colombo JS, Jia S, D’Souza RN (2020) Modeling hypoxia induced factors to treat pulpal inflammation and drive regeneration. J Endod 46: S19-S25.

Colombo JS, Moore AN, Hartgerink JD, D'Souza RN (2014) Scaffolds to control inflammation and facilitate dental pulp regeneration. J Endod 40: S6-S12.

Cooper PR, Takahashi Y, Graham LW, Simon S, Imazato S, Smith AJ (2010) Inflammationregeneration interplay in the dentine-pulp complex. J Dent 38: 687-697.

Cruse WP, Bellizzi R (1980) A historic review of endodontics, 1689-1963, part 1. J Endod 6: 495-499.

Duncan HF, Cooper PR (2020) Pulp innate immune defense: Translational opportunities. J Endod 46: S10-S18.

Eramo S, Natali A, Pinna R, Milia E (2018) Dental pulp regeneration via cell homing. Int Endod J 51: 405-419.

Farges JC, Alliot-Licht B, Renard E, Ducret M, Gaudin A, Smith AJ, Cooper PR (2015) Dental pulp defence and repair mechanisms in dental caries. Mediators Inflamm 2015: 230251. DOI: $10.1155 / 2015 / 230251$.

Frantz C, Stewart KM, Weaver VM (2010) The extracellular matrix at a glance. J Cell Sci 123: 41954200 .

Friedman S, Mor C (2004) The success of endodontic therapy-healing and functionality. J Calif Dent Assoc 32: 493-503.

Giraud T, Jeanneau C, Rombouts C, Bakhtiar H, Laurent P, About I (2019) Pulp capping materials modulate the balance between inflammation and regeneration. Dent Mater 35: 24-35.

Goldberg M, Farges JC, Lacerda-Pinheiro S, Six N, Jegat N, Decup F, Septier D, Carrouel F, Durand $S$, Chaussain-Miller C, Denbesten P, Veis A, Poliard 
A (2008) Inflammatory and immunological aspects of dental pulp repair. Pharmacol Res 58: 137-147.

Gordon S (2003) Alternative activation of macrophages. Nat Rev Immunol 3: 23-35.

Gordon S, Martinez FO (2010) Alternative activation of macrophages: Mechanism and functions. Immunity 32: 593-604.

Gordon S, Taylor PR (2005) Monocyte and macrophage heterogeneity. Nat Rev Immunol 5: 953964.

Hahn CL, Liewehr FR (2007) Relationships between caries bacteria, host responses, and clinical signs and symptoms of pulpitis. J Endod 33: 213-219.

Heng C (2016) Tooth decay is the most prevalent disease. Fed Pract 33: 31-33.

Huang GT, Liu J, Zhu X, Yu Z, Li D, Chen CA, Azim AA (2020) Pulp/dentin regeneration: it should be complicated. J Endod 46: S128-S134.

Ibuki T, Kido MA, Kiyoshima T, Terada Y, Tanaka T (1996) An ultrastructural study of the relationship between sensory trigeminal nerves and odontoblasts in rat dentin/pulp as demonstrated by the anterograde transport of wheat germ agglutininhorseradish peroxidase (wga-hrp). J Dent Res 75: 1963-1970.

Iohara K, Zayed M, Takei Y, Watanabe H, Nakashima M (2020) Treatment of pulpectomized teeth with trypsin prior to transplantation of mobilized dental pulp stem cells enhances pulp regeneration in aged dogs. Front Bioeng Biotechnol 8: 983 . DOI: $10.3389 /$ fbioe.2020.00983.

Iohara K, Zheng L, Ito M, Ishizaka R, Nakamura $\mathrm{H}$, Into T, Matsushita K, Nakashima M (2009) Regeneration of dental pulp after pulpotomy by transplantation of cd31(-)/cd146(-) side population cells from a canine tooth. Regen Med 4: 377-385.

Kawashima N, Nakano-Kawanishi H, Suzuki N, Takagi M, Suda H (2005) Effect of nos inhibitor on cytokine and COX2 expression in rat pulpitis. J Dent Res 84: 762-767.

Keller L, Offner D, Schwinte P, Morand D, Wagner Q, Gros C, Bornert F, Bahi S, Musset AM, BenkiraneJessel N, Fioretti F (2015) Active nanomaterials to meet the challenge of dental pulp regeneration. Materials (Basel) 8: 7461-7471.

Kline LW, Yu DC (2009) Effects of calcitonin, calcitonin gene-related peptide, human recombinant bone morphogenetic protein-2, and parathyroid hormone-related protein on endodontically treated ferret canines. J Endod 35: 866-869.

Laird DJ, von Andrian UH, Wagers AJ (2008) Stem cell trafficking in tissue development, growth, and disease. Cell 132: 612-630.

Lara VS, Figueiredo F, da Silva TA, Cunha FQ (2003) Dentin-induced in vivo inflammatory response and in vitro activation of murine macrophages. J Dent Res 82: 460-465.

Le Fournis C, Hadjichristou C, Jeanneau C, About I (2019) Human pulp fibroblast implication in phagocytosis via complement activation. J Endod 45: 584-590.
Lehwald N, Duhme C, Pinchuk I, Kirchner J, Wieferich K, Schmelzle M, Jurk K, Windmoller BA, Hubner W, Homey B, Bode J, Kubitz R, Benhidjeb T, Krüger M, Robson SC, Knoefel WT, Kehrel BE, Schulte Am Esch J (2020) Platelets boost recruitment of CD133(+) bone marrow stem cells to endothelium and the rodent liver-the role of P-selectin/PSGL-1 interactions. Int J Mol Sci 21: 6341. DOI: 10.3390/ ijms21176431.

Li Y, Sui B, Dahl C, Bergeron B, Shipman P, Niu L, Chen J, Tay FR (2019) Pulpotomy for carious pulp exposures in permanent teeth: a systematic review and meta-analysis. J Dent 84: 1-8.

Lucarotti PSK (2003) The life expectancy of dental restorations placed within the general dental services in England and Wales. PhD dissertation. University of Birmingham.

Mahmoudi J, Sabermarouf B, Baradaran B, SadatHatamnezhad L, Shotorbani SS (2017) Up-regulation of TLR2 and TLR4 in high mobility group Box1stimulated macrophages in pulpitis patients. Iran J Basic Med Sci 20: 209-215.

Mantovani A, Sica A, Sozzani S, Allavena P, Vecchi A, Locati M (2004) The chemokine system in diverse forms of macrophage activation and polarization. Trends Immunol 25: 677-686.

Markley MR (1951) Restorations of silver amalgam. J Am Dent Assoc 43: 133-146.

Mitsiadis TA, Magloire H, Pagella P (2017) Nerve growth factor signalling in pathology and regeneration of human teeth. Sci Rep 7: 1327. DOI: 10.1038/s41598-017-01455-3.

Mutoh N, Watabe H, Chieda K, Tani-Ishii N (2009) Expression of toll-like receptor 2 and 4 in inflamed pulp in severe combined immunodeficiency mice. J Endod 35: 975-980.

Naito T (2005) Better success rate for root canal therapy when treatment includes obturation short of the apex. Evid Based Dent 6: 45. DOI: 10.1038/ sj.ebd.6400335.

Nakashima M, Iohara K, Murakami M, Nakamura H, Sato Y, Ariji Y, Matsushita K (2017) Pulp regeneration by transplantation of dental pulp stem cells in pulpitis: a pilot clinical study. Stem Cell Res Ther 8: 61. DOI: 10.1186/s13287-017-0506-5.

Radke D, Jia W, Sharma D, Fena K, Wang G, Goldman J, Zhao F (2018) Tissue engineering at the blood-contacting surface: a review of challenges and strategies in vascular graft development. Adv Healthc Mater 7: e1701461. DOI: 10.1002/ adhm.201701461.

Ray HA, Trope M (1995) Periapical status of endodontically treated teeth in relation to the technical quality of the root filling and the coronal restoration. Int Endod J 28: 12-18.

Ray HL Jr, Marcelino J, Braga R, Horwat R, Lisien M, Khaliq S (2016) Long-term follow up of revascularization using platelet-rich fibrin. Dent Traumatol 32: 80-84.

Roy S (2016) Mirna in macrophage development and function. Antioxid Redox Signal 25: 795-804. 
Sadaf D (2020) Success of coronal pulpotomy in permanent teeth with irreversible pulpitis: an evidence-based review. Cureus 12: e6747. DOI: 10.7759/cureus.6747.

Salehrabi R, Rotstein I (2004) Endodontic treatment outcomes in a large patient population in the USA: an epidemiological study. J Endod 30: 846850.

Shimeno Y, Sugawara Y, Iikubo M, Shoji N, Sasano $\mathrm{T}$ (2008) Sympathetic nerve fibers sprout into rat odontoblast layer, but not into dentinal tubules, in response to cavity preparation. Neurosci Lett 435: 73-77.

Sicari BM, Dziki JL, Siu BF, Medberry CJ, Dearth CL, Badylak SF (2014) The promotion of a constructive macrophage phenotype by solubilized extracellular matrix. Biomaterials 35: 8605-8612.

Siqueira JF Jr (2001) Aetiology of root canal treatment failure: why well-treated teeth can fail. Int Endod J 34: 1-10.

Soares DG, Zhang Z, Mohamed F, Eyster TW, de Souza Costa CA, Ma PX (2018) Simvastatin and nanofibrous poly(l-lactic acid) scaffolds to promote the odontogenic potential of dental pulp cells in an inflammatory environment. Acta Biomater 68: 190203.

Souron JB, Petiet A, Decup F, Tran XV, Lesieur J, Poliard A, Le Guludec D, Letourneur D, Chaussain C, Rouzet F, Vital SO (2014) Pulp cell tracking by radionuclide imaging for dental tissue engineering. Tissue Eng Part C Methods 20: 188-197.

St Pierre BA, Tidball JG (1994) Differential response of macrophage subpopulations to soleus muscle reloading after rat hindlimb suspension. J Appl Physiol (1985) 77: 290-297.

Staffoli S, Plotino G, Nunez Torrijos BG, Grande NM, Bossu M, Gambarini G, Polimeni A (2019) Regenerative endodontic procedures using contemporary endodontic materials. Materials (Basel) 12: 908. DOI: $10.3390 / \mathrm{ma1} 2060908$.

Swirski FK, Nahrendorf M, Etzrodt M, Wildgruber M, Cortez-Retamozo V, Panizzi P, Figueiredo JL, Kohler RH, Chudnovskiy A, Waterman P, Aikawa E, Mempel TR, Libby P, Weissleder R, Pittet MJ (2009) Identification of splenic reservoir monocytes and their deployment to inflammatory sites. Science 325 612-616.

Tidball JG (2005) Inflammatory processes in muscle injury and repair. Am J Physiol Regul Integr Comp Physiol 288: R345-353.

Tidball JG, Wehling-Henricks M (2007) Macrophages promote muscle membrane repair and muscle fibre growth and regeneration during modified muscle loading in mice in vivo. J Physiol 578: 327-336.
Valentin JE, Stewart-Akers AM, Gilbert TW, Badylak SF (2009) Macrophage participation in the degradation and remodeling of extracellular matrix scaffolds. Tissue Eng Part A 15: 1687-1694.

Villalta SA, Nguyen HX, Deng B, Gotoh T, Tidball JG (2009) Shifts in macrophage phenotypes and macrophage competition for arginine metabolism affect the severity of muscle pathology in muscular dystrophy. Hum Mol Genet 18: 482-496.

Vongsavan N, Matthews RW, Matthews B. 2000. The permeability of human dentine in vitro and in vivo. Arch Oral Biol 45: 931-935.

Wells C, Dulong C, McCormack S (2019) Vital pulp therapy for endodontic treatment of mature teeth: a review of clinical effectiveness, cost-effectiveness, and guidelines. CADTH Rapid Response Reports. Ottawa $(\mathrm{ON})$.

Woodnutt DA, Wager-Miller J, O'Neill PC, Bothwell M, Byers MR (2000) Neurotrophin receptors and nerve growth factor are differentially expressed in adjacent nonneuronal cells of normal and injured tooth pulp. Cell Tissue Res 299: 225-236.

Yonehiro J, Yamashita A, Yoshida Y, Yoshizawa S, Ohta K, Kamata N, Okihara T, Nishimura F (2012) Establishment of an ex vivo pulpitis model by co-culturing immortalized dental pulp cells and macrophages. Int Endod J 45: 1103-1108.

Yoshiba N, Edanami N, Ohkura N, Maekawa T, Takahashi N, Tohma A, Izumi K, Maeda T, Hosoya A, Nakamura H, Tabeta K, Noiri Y, Yoshiba K (2020) M2 phenotype macrophages colocalize with schwann cells in human dental pulp. J Dent Res 99: 329-338.

Zaky SH, AlQahtani Q, Chen J, Patil A, Taboas J, Beniash E, Ray H, Sfeir C (2020) Effect of the periapical "inflammatory plug" on dental pulp regeneration: a histologic in vivo study. J Endod 46: 51-56.

Zaky SH, Cancedda R (2009) Engineering craniofacial structures: Facing the challenge. J Dent Res 88: 1077-1091.

Zhan C, Huang M, Yang X, Hou J (2020) Dental nerves: A neglected mediator of pulpitis. Int Endod J 99: 329-338.

Zhuang Z, Yoshizawa-Smith S, Glowacki A, Maltos K, Pacheco C, Shehabeldin M, Mulkeen M, Myers N, Chong R, Verdelis K,Garlet GP, Little S, Sfeir C (2019) Induction of M2 macrophages prevents bone loss in murine periodontitis models. J Dent Res 98: 200-208.

Editor's note: All questions from reviewers were answered in the text, therefore there is no Discussion with Reviewers section for this manuscript. The Scientific Editor responsible for this paper was Thimios Mitsiadis. 